\title{
Substrates coated with silver nanoparticles as a neuronal regenerative material
}

\author{
This article was published in the following Dove Press journal: \\ International Journal of Nanomedicine \\ 8 May 2014 \\ Number of times this article has been viewed
}

\author{
Noa Alon ${ }^{1,3, *}$ \\ Yana Miroshnikov ${ }^{2,3, *}$ \\ Nina Perkas ${ }^{2,3}$ \\ Ifat Nissan ${ }^{2,3}$ \\ Aharon Gedanken ${ }^{2,3}$ \\ Orit Shefi ${ }^{1,3}$ \\ 'Faculty of Engineering, ${ }^{2}$ Department \\ of Chemistry, ${ }^{3}$ Bar-llan Institute \\ of Nanotechnology and Advanced \\ Materials, Bar-Ilan University, Ramat \\ Gan, Israel \\ *These authors contributed equally to \\ this work
}

Correspondence: Orit Shefi Faculty of Engineering, Bar llan University, Ramat Gan, 52900, Israel

Tel +972 35317079

Fax+972 37384646

Email orit.shefi@biu.ac.il
Abstract: Much effort has been devoted to the design of effective biomaterials for nerve regeneration. Here, we report the novel use of silver nanoparticles (AgNPs) as regenerative agents to promote neuronal growth. We grew neuroblastoma cells on surfaces coated with AgNPs and studied the effect on the development of the neurites during the initiation and the elongation growth phases. We find that the AgNPs function as favorable anchoring sites, and the growth on the AgNP-coated substrates leads to a significantly enhanced neurite outgrowth. Cells grown on substrates coated with AgNPs have initiated three times more neurites than cells grown on uncoated substrates, and two times more than cells grown on substrates sputtered with a plain homogenous layer of silver. The growth of neurites on AgNPs in the elongation phase was enhanced as well. A comparison with substrates coated with gold nanoparticles (AuNPs) and zinc oxide nanoparticles (ZnONPs) demonstrated a clear silver material-driven promoting effect, in addition to the nanotopography. The growth on substrates coated with AgNPs has led to a significantly higher number of initiating neurites when compared to substrates coated with AuNPs or ZnONPs. All nanoparticle-coated substrates affected and promoted the elongation of neurites, with a significant positive maximal effect for the AgNPs. Our results, combined with the well-known antibacterial effect of AgNPs, suggest the use of AgNPs as an attractive nanomaterial - with dual activity - for neuronal repair studies.

Keywords: nerve regeneration, nanotopography, antibacterial material, neuroblastoma, gold nanoparticles, zinc oxide nanoparticles

\section{Introduction}

The manipulation of neuronal growth has important applications in regenerative biomedicine and bioengineering. Neurons grow and function while sensing and responding to a wide range of chemical and physical environmental signals through the tips of their developing neurites. The cells are highly influenced by the properties of the growth substrates. ${ }^{1-4}$ It has been recognized that structured surfaces are more favorable for axonal growth than smooth ones. ${ }^{5,6}$ Recent studies demonstrate that topographic cues - even at the nanoscale - influence neuronal growth. ${ }^{7-10}$ Nanowires have been utilized to guide axons, ${ }^{11}$ and nanopillars have been used to position neurons by serving as geometrical adhesion sites. ${ }^{12}$ We have recently shown that neuronal growth is affected and directed by nanoscale line-pattern ridges. ${ }^{13,14}$ The interactions of neurons with the nanotopographic cues have led to an elongation of neuronal branching length while showing no positive effect on the neurites' initiation. Brunetti et a ${ }^{15}$ have demonstrated that neuroblastoma cells sense and respond to surface nanotopography with a sensitivity of a few nanometers. In these studies, the effect on the neuronal 
growth is due to the surface nanotopography. Here, we will study the combination of physical topographical stimulation together with chemical stimulation by using active materials as the nanotopographical cues. We will use silver nanoparticles (AgNPs) that are well-known for their antibacterial activity. ${ }^{16-20} \mathrm{We}$ will compare their effect to zinc oxide nanoparticles (ZnONPs), which also possess antibacterial properties, ${ }^{21,22}$ and to gold nanoparticles (AuNPs), which are considered inert. Although AgNPs were reported to have a cytotoxic effect on cells, ${ }^{23-25}$ and their use in medical applications is not widespread, recent studies reveal that AgNPs can be biocompatible. ${ }^{26}$ Ragaseema et $\mathrm{al}^{27}$ have demonstrated antibacterial, antiplatelet, and noncytotoxic properties of AgNPs for the use in cardiovascular implantable devices. Fullenkamp et $a^{28}$ developed silver (Ag)-releasing hydrogels, which showed no effect on the viability of mammalian cell lines. Several studies have even reported on the possible pro-healing properties of AgNPs..$^{29-31}$

We will use the sonochemical method ${ }^{19,32}$ to fabricate surfaces coated with the AgNPs, ZnONPs, or AgNPs at controlled sizes and concentrations. In the current study, we will investigate the interactions between the coated substrates and the human neuroblastoma cells (SH-SY5Y) that show high sensitivity to environmental stimuli. ${ }^{33} \mathrm{We}$ will examine the effects of the different materials on the pattern of growth. We will also quantify the morphology of the cells along their course of development during the first regenerative phase of neurite initiation (where branches emerge out of the soma), and during the second phase of neurite elongation.

We found that cells grown on substrates coated with AgNPs have significantly developed more neurites than cells grown on substrates coated with AuNPs or ZnONPs. Cells grown on substrates coated with a homogeneous layer of Ag, with no topographical stimulation, have also demonstrated a promoted effect at the neurites' initiation growth phase. During the neurites' elongation phase, all nanotopographical cues of all materials have led to a promoted growth - yet again - with a maximal effect of the AgNPs.

Our results demonstrate the AgNPs as an active neuronal regenerative material, combining nanotopography and a material-driven effect. The positive influence on neuronal growth, together with the known antibacterial properties of AgNPs, proposes substrates coated with AgNPs as an attractive therapeutic platform. Our fabrication-coating methodology that may be implemented for coating three-dimensional scaffolds opens new transformative research directions for the design of transplanted biomedical devices.

\section{Materials and methods}

\section{Substrate fabrication and characterization}

We have fabricated substrates coated with AgNPs by using the sonochemical method, as described previously by Perkas et al. ${ }^{20}$ This technique is a one-step process, in which the nanoparticles (NPs) are fabricated and subsequently thrown onto the glass substrate at a very high speed so that they are well embedded into the glass. Briefly, a $1 \times 0.8 \mathrm{~cm}^{2}$ glass slide was positioned in a sonication cell containing $100 \mathrm{~mL}$ of water-ethylene glycol (10 vol\% ethylene glycol) and $0.05 \mathrm{M}$ of $\mathrm{Ag}$ nitrate solution. The solution was added to the sonication cell and purged under a flow of argon for 30 minutes. The solution was then irradiated with a high-intensity ultrasonic horn (Ti horn, $20 \mathrm{kHz}, \mathrm{VCX} 600$ sonifier, Sonics and Materials [Newtown, CT, USA]) for 8 minutes. Substrates coated with AuNPs and ZnONPs were fabricated by using the same method (see Table S1). We use uncoated glass substrates and glass substrates sputtered with a homogeneous layer of $\mathrm{Ag}$ or gold (80-200 nm thick) as controls. Parameters of particle size and particle density were obtained using the ImageJ software (National Institutes of Health, Bethesda, MD, USA), based on the high-resolution scanning electron microscope (HRSEM) images.

\section{Cell culture}

The SH-SY5Y human neuroblastoma cells were cultured in a humidified incubator at $37^{\circ} \mathrm{C}$ containing $5 \% \mathrm{CO}_{2}$ and were routinely grown in $75 \mathrm{~cm}^{2}$ plastic tissue-culture flasks containing $10 \mathrm{~mL}$ of Dulbecco's Modified Eagle's Medium, supplemented with $2 \mathrm{mM}$ L-glutamine, 5\% penicillinstreptomycin, $1 \%$ amphotericin, and $10 \%$ heat-inactivated fetal bovine serum. The medium was replaced every $2-3$ days, and the cells were split by adding $5 \mathrm{~mL}$ trypsin when reaching a confluence of $\sim 80 \%$. Coated and uncoated substrates were sterilized under ultraviolet light for 30 minutes, and each substrate was placed in a $35 \mathrm{~mm}$ dish before plating the cells. Cells were plated in an initial concentration of $10^{5}$ cells per dish and cultured under the same conditions as described previously. We followed the cell cultures daily for up to a week using light microscopy (Leica DM IL LED; Leica Microsystems, Wetzlar, Germany). Images were acquired using a Leica DFC290 HD camera (Leica Microsystems).

\section{Viability test}

An Annexin V-FITC (fluorescein isothiocyanate) apoptosis kit (MBL International Corporation, Woburn, MA, USA) was used for a direct count of viable, apoptotic, and necrotic cells. The SH-SY5Y cells were grown on substrates coated with 
AgNPs and control on uncoated glass, as described above. After 24 hours, the cells were washed once with phosphate buffered saline (PBS) and then resuspended in a binding buffer. Annexin V-FITC and propidium iodide were added, according to the manufacturer's instructions, and the samples were incubated in the dark for 15 minutes. For cell counting, images were acquired by a confocal microscope (Leica SP5-II; Leica Microsystems) and were then analyzed manually by using the cell counter plugin in the ImageJ software.

\section{Scanning electron microscopy}

Two days after plating, the cells were fixed with $2 \%$ glutaraldehyde in PBS (no $\mathrm{Ca}^{3+}$; no $\mathrm{Mg}^{3+} ; \mathrm{pH}, 7.2$ ) for 1 hour at room temperature. After fixation, the samples were repeatedly rinsed in PBS and then treated with a $2 \%$ guanidinehydrochloride:tannic acid (4:5) solution for 1 hour at room temperature. Samples were repeatedly rinsed again in PBS. Then, they were dehydrated, using increasing concentrations of ethanol $(50 \%, 70 \%, 80 \%, 90 \%$, and $100 \%)$ and increasing concentrations of Freon ${ }^{\circledR}$ (DuPont ${ }^{\mathrm{TM}}$, Wilmington, DE, USA) $(50 \%, 75 \%, 100 \% \times 3)$. Finally, the samples were sputtered with carbon before examination in the Magellan 400L HRSEM (FEI, Hillsboro, OR, USA).

\section{Morphological and statistical analysis}

The HRSEM images were used for morphological analysis. The number of neurites emerging from the soma and the number of neurites at a radius of $5 \mu \mathrm{m}$ from the soma were measured using the NeuronJ tool ${ }^{34}$ (ImageJ software). To obtain linear fits and the $R^{2}$ values of neurites per cell, we used NeuronJ for tracing, and the $\mathrm{X}$ and $\mathrm{Y}$ coordinates of the neurites were then analyzed using an in-house MATLAB (The Mathworks, Inc, Natick, MA, USA) script. A minimum of 50 $\mathrm{X}$ and $\mathrm{Y}$ values per neurite were taken. Statistical significance was determined using the Mann-Whitney $U$ test with the Benjamini-Hochberg method and an adjusted $P$-value.

\section{Results}

We fabricated substrates coated with AgNPs, ZnONPs, and AuNPs at controlled average densities and size. Figure 1 shows scanning electron microscope (SEM) images of substrates coated with the three types of NPs, demonstrating the similar average densities and particle diameters (see Materials and methods and Table 1).

We first examined the effect of the NPs on cell viability. The morphology and development of the SH-SY5Y cells grown on AgNPs, ZnONPs, and AuNPs appeared normal under light microscopy observations, similar to the cells grown on glass substrates (Figure 2). To assess cell death on the AgNP-coated substrates, we used an Annexin V-FITC and propidium iodide staining assay. On both AgNP-coated substrates and uncoated control substrates, only a small percentage of cells underwent apoptosis or necrosis. As can be seen in Figure 3, similar results were obtained for cells grown on the AgNP-coated substrates and on the control substrates. We found that $1.7 \% \pm 0.4 \%$ of the cells on the AgNPs and $1.6 \% \pm 0.2 \%$ of the cells on the control substrates were apoptotic. We also found that $2.6 \% \pm 0.3 \%$ of the cells on the AgNP-coated substrates and $2.7 \% \pm 0.4 \%$ of the cells on the control substrates were necrotic.

Next, we examined the growth of the cells on the substrates coated with AgNPs, AuNPs, or ZnONPs by using a HRSEM. We used uncoated substrates and substrates sputtered with the homogeneous layer of $\mathrm{Ag}$ (AgSput) or gold (AuSput), with no topographical pattern, as controls. Figure 4 presents typical cells on the studied substrates. The HRSEM images demonstrate an increased number of the neurites for cells grown on the AgNPs. To quantify this effect on the neurites' initiation and development, we measured the number of neurites emerging from the soma per cell and the number of neurites in a radius of $5 \mu \mathrm{m}$ from the soma per cell (Figure 5A). The mean number of neurites emerged from the cells grown on the AgNP-coated
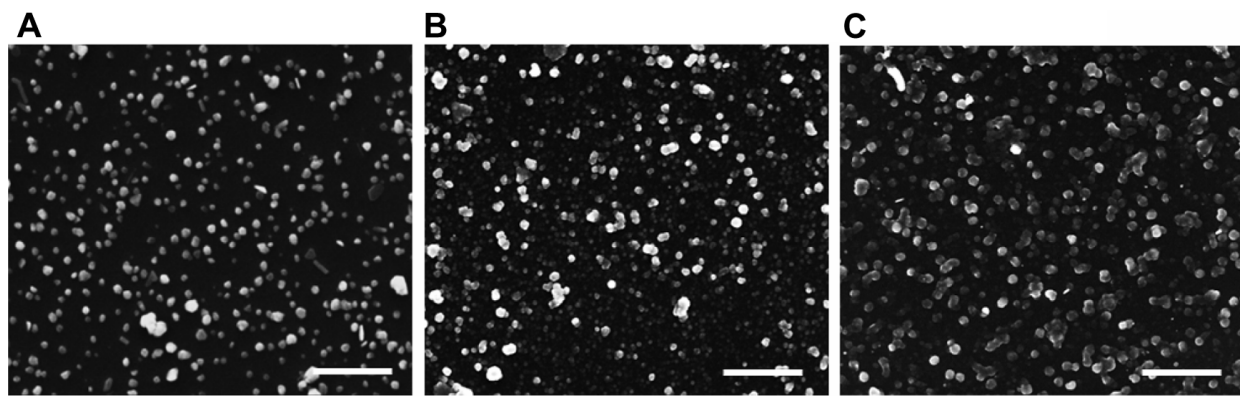

Figure I SEM images of substrates.

Notes: SEM images of substrates coated with (A) AgNPs, (B) AuNPs, and (C) ZnONPs. Scale bar, I $\mu \mathrm{m}$

Abbreviations: SEM, scanning electron microscope; AgNPs, silver nanoparticles; AuNPs, gold nanoparticles; ZnONPs, zinc oxide nanoparticles. 
Table I Particle size and density for the NP-coated substrates

\begin{tabular}{lll}
\hline Substrate & $\begin{array}{l}\text { Particle } \\
\text { diameter }(\mathbf{n m})\end{array}$ & $\begin{array}{l}\text { Particle density } \\
\left(\text { particles } / \mu \mathbf{m}^{2}\right)\end{array}$ \\
\hline AgNP coating & $110 \pm 40$ & $14 \pm 1$ \\
AuNP coating & $100 \pm 40$ & $16 \pm 5$ \\
ZnONP coating & $115 \pm 45$ & $15 \pm 4$ \\
\hline
\end{tabular}

Abbreviations: NP, nanoparticle; AgNP, silver nanoparticle; AuNP, gold nanoparticle; $\mathrm{ZnONP}$, zinc oxide nanoparticle.

substrates was significantly enhanced in comparison to the glass substrates $(2.8 \pm 0.2$ times more; $P<0.001)$ and to the $\mathrm{Ag}$-sputtered substrates ( $1.8 \pm 0.1$ times more; $P<0.01)$, as shown in Figure 5B. Figure 5B also demonstrates that cells grown on the Ag-sputtered substrates have developed more neurites when compared to the uncoated glass substrates $(P<0.001)$. When measured at a distance of $5 \mu \mathrm{m}$ from the soma, the number of neurites counted in cells grown on the AgNP substrates was $2.6 \pm 0.1$ times more than on the glass substrates $(P<0.001)$; the number of neurites on the Ag-sputtered substrates was similar to the glass substrates, with $0.9 \pm 0.1$ similarity (Figure 5C).

Figure 5B presents the number of neurites emerging from the soma of cells grown on AuNP- and ZnONP-coated substrates, in addition to the AgNP substrates. It can be seen that the number of neurites on the NP substrates, normalized to glass, is significantly higher for the AgNPs in comparison to the AuNPs $(2.8 \pm 0.2$ and $1.2 \pm 0.1$ times more, respectively; $P<0.001)$ and to the ZnONPs $(1.1 \pm 0.1$ times more; $P<0.001)$. The comparison of the number of neurites on these substrates that were $5 \mu \mathrm{m}$ from the soma demonstrates a
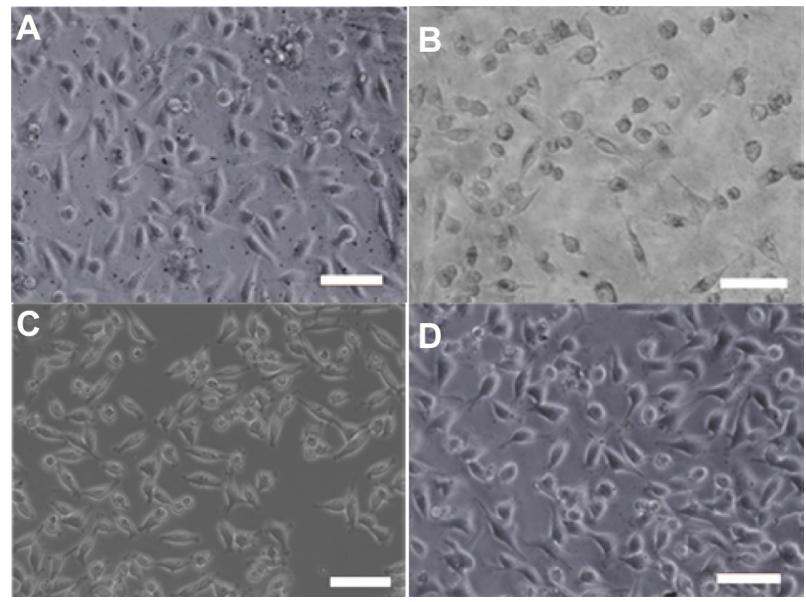

Figure 2 Light microscopy images of SH-SY5Y cells.

Notes: These images present cells that were grown on substrates coated with (A) AgNPs, (B) AuNPs, (C) ZnONPs, and (D) on an uncoated glass substrate. Scale bar $=50 \mu \mathrm{m}$. Abbreviations: AgNPs, silver nanoparticles; AuNPs, gold nanoparticles; ZnONPs, zinc oxide nanoparticles.

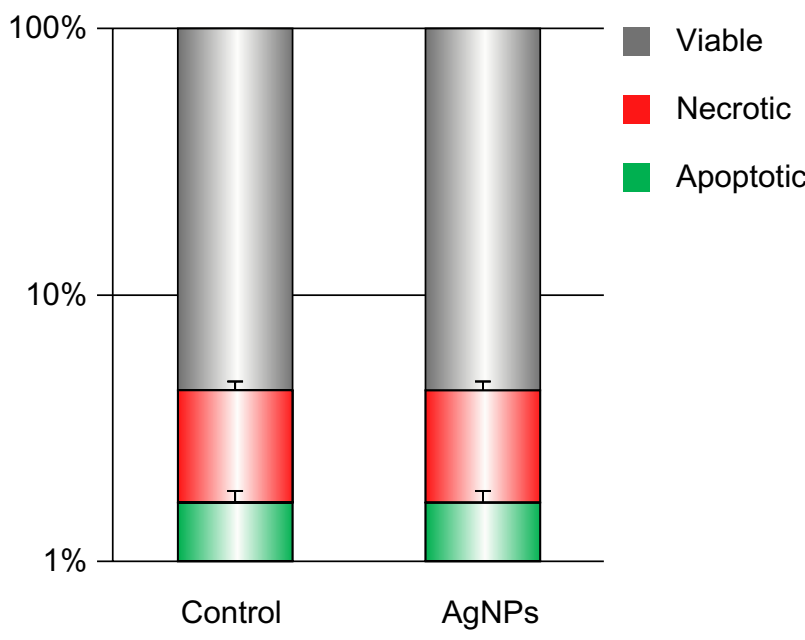

Figure 3 Annexin V-FITC and propidium iodide staining for the detection of apoptotic and necrotic cells.

Notes: The majority of the cells on both types of substrates were found to be viable. Less than $2 \%$ of the cells on both the control and the AgNP substrates were apoptotic, and less than $3 \%$ of the cells on both types of substrates were necrotic. The $Y$-axis is presented in a logarithmic scale.

Abbreviations: AgNPs, silver nanoparticles; FITC, fluorescein isothiocyanate.
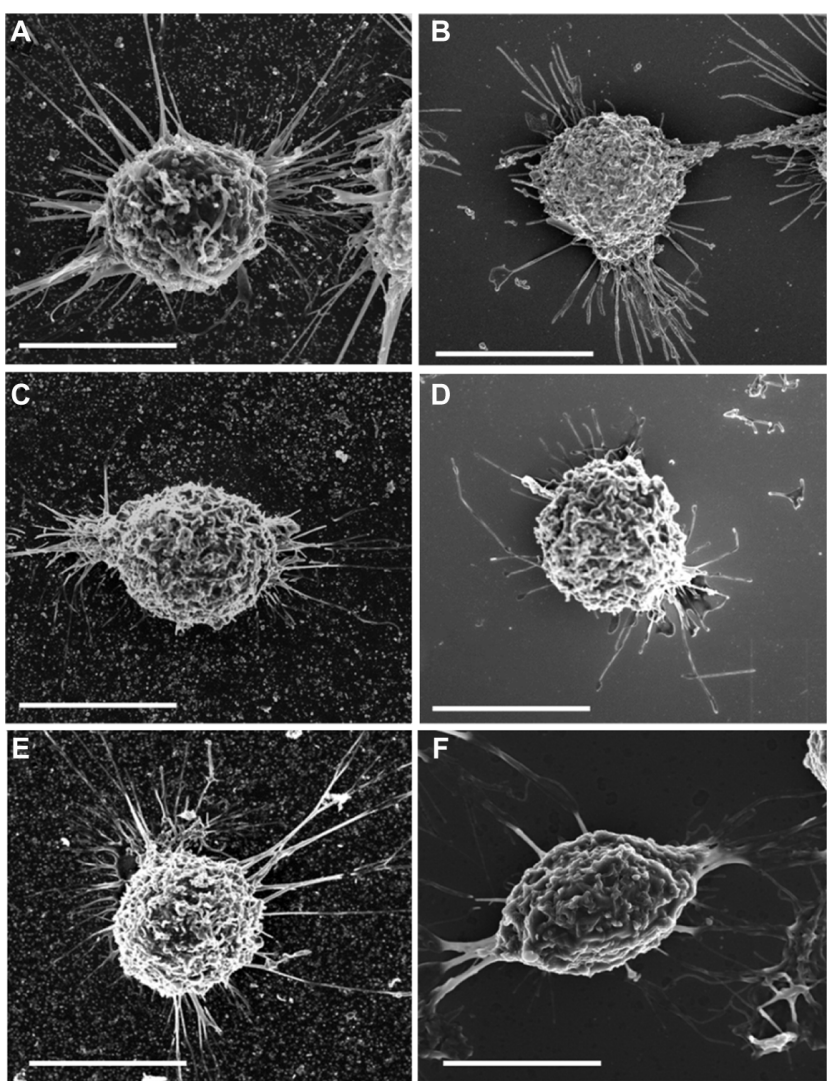

Figure 4 HRSEM images of representative cells.

Notes: These HRSEM images show representative cells grown on substrates coated with (A) AgNPs, (B) a homogeneous layer of Ag, (C) AuNPs, (D) a homogeneous layer of $\mathrm{Au},(\mathrm{E}) \mathrm{ZnONPs}$, and $(\mathbf{F})$ on an uncoated glass substrate. Scale bar $=10 \mu \mathrm{m}$. Abbreviations: HRSEM, high-resolution scanning electron microscopy; AgNPs, silver nanoparticles; Ag, silver; AuNPs, gold nanoparticles; Au, gold; ZnONPs, zinc oxide nanoparticles. 
A
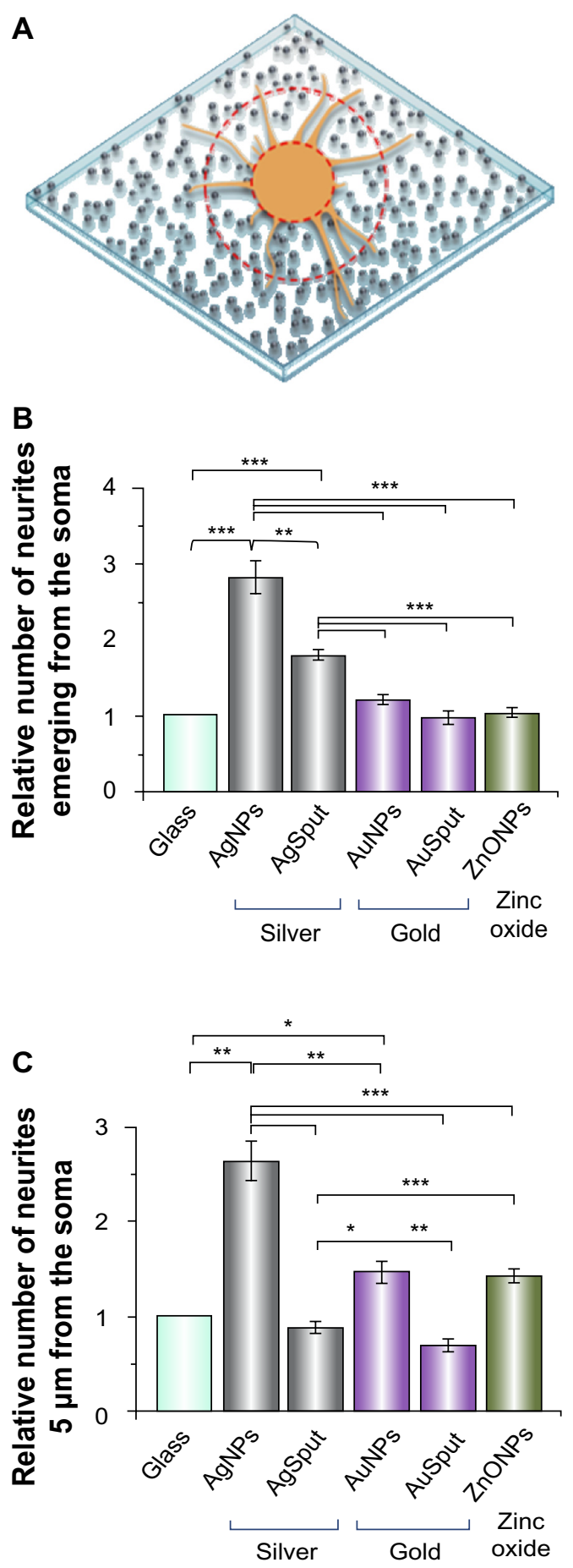

Figure 5 Measurements of the number of neurites.

Notes: (A) A schematic illustration of a cell grown on the AgNP-coated substrate. The locations at which the neurites were counted are represented by the inner and outer red circles, close to the soma and $5 \mu \mathrm{m}$ from the soma, respectively. (B) A comparison of the relative number of neurites emerging from the soma between glass and the AgNP, AuNP, and ZnONP substrates, as well as between the AgSput and AuSput. (C) A comparison of the relative number of neurites, $5 \mu \mathrm{m}$ from the soma, between the AgNP, AuNP, and ZnONP substrates, as well as between the AgSput and AuSput. Data is presented as the mean \pm SE normalized to glass substrates, $n=8\left({ }^{*} P<0.05\right.$; $* * P<0.0$ I; ${ }^{* * *} P<0.00$ I, Mann-Whitney $U$ test with Benjamini-Hochberg adjusted $P$-values).

Abbreviations: AgNPs, silver nanoparticles; AgSput, silver sputtering; AuSput, gold sputtering; ZnONPs, zinc oxide nanoparticles; SE, standard error; n, number. similar trend, presenting a maximal promoting growth effect for cells on the AgNPs substrates (Figure 5C). Cells on the AuNPs and the ZnONPs substrates show a larger number of neurites per cell than on glass $(1.5 \pm 0.1$ and $1.4 \pm 0.1$, respectively; $P<0.05$ for the AuNPs). Cells grown on the AuSput substrates showed similar statistics as on the glass, in contradiction to the Ag.

To closely examine the effect of the NPs on cell morphology, we have analyzed the interactions between the cells to AgNPs that have demonstrated maximal effect on growth. Figure 6 presents the significant effect of the AgNPs on neurite outgrowth, as observed under HRSEM. Neurites were found to be highly straightened in comparison to
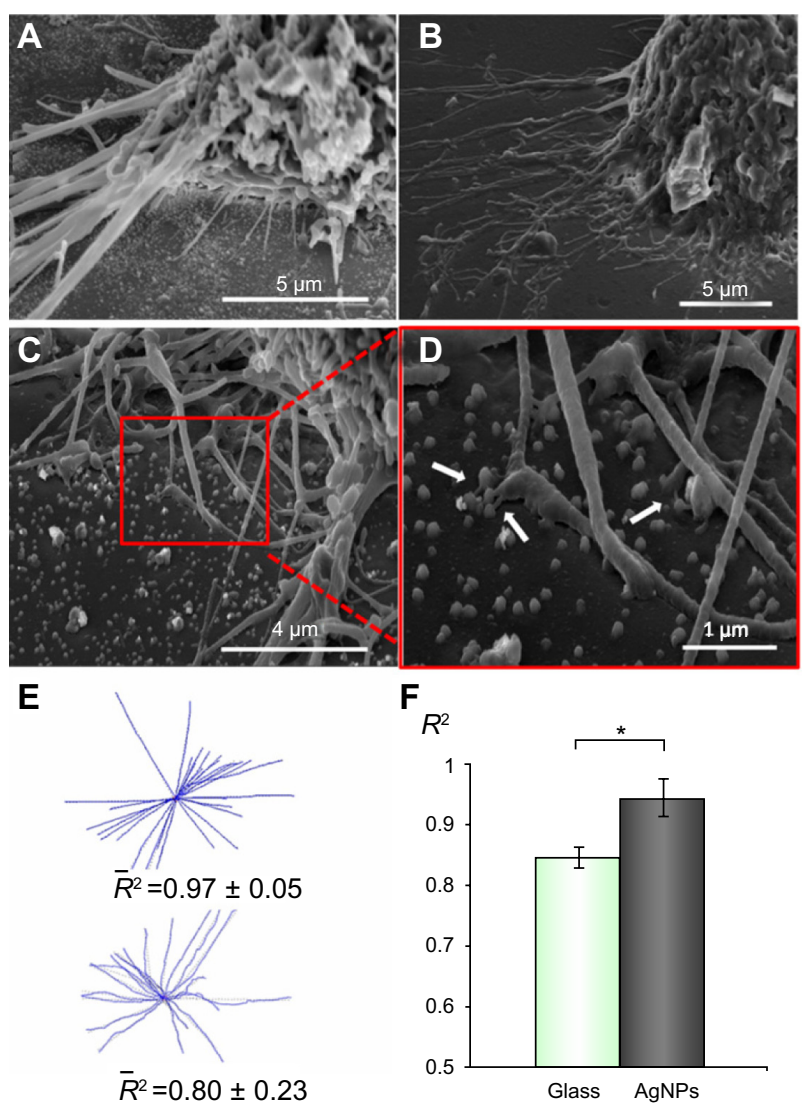

Figure 6 HRSEM images demonstrate the effect of AgNPs on neurite outgrowth. Notes: (A) A typical cell grown on an AgNP-coated substrate grows highly straightened neurites in comparison to (B) a cell grown on an uncoated control substrate. (C) Neurites of a cell grown on the AgNP-coated substrate emerging from the cell body and attaching to the AgNPs. (D) The zoomed-in image of (C) reveals nanoscale extensions of the neurites attached to the NPs (white arrows). (E and F) A quantitative analysis of the neurites' morphology. (E) Neurite tracing, their corresponding linear fits, and their average $R^{2}$ values are shown for the representative cell grown on the AgNP-coated substrate and on a control glass substrate. (F) Average $R^{2}$ values of neurites emerging from cells grown on AgNPcoated substrates are significantly higher than the cells grown on control glass substrates (mean $\pm \mathrm{SE}, \mathrm{n}=6, * \mathrm{P}<0.0 \mathrm{l}$ ).

Abbreviations: HRSEM, high-resolution scanning electron microscopy; NPs, nanoparticles; AgNPs, silver nanoparticles; SE, standard error; n, number. 
cells grown on control glass substrates that develop curved branches (Figure 6A and B). High-magnification images revealed the nanoscale extensions emerging from the neurites and contacting the NPs (Figures 6C and D). To quantify the effect of the AgNPs on the morphology of the neurites, we obtained linear fits and $R^{2}$ values of neurites per cell (Figure 6E and F). Higher $R^{2}$ values indicate neurites that are closer to straight lines. We have found significantly higher $R^{2}$ values, $0.94 \pm 0.02$ on average, for neurites atop coated substrates, while growth on the glass substrates resulted in lower $R^{2}$ values ( $0.84 \pm 0.03$ in average), as shown in Figure 6F. It is important to note that many branches of the control cells were too curved to be traced and were not included in the statistical analysis.

\section{Discussion}

This study demonstrates the role of AgNPs as a preferable regenerative substance combining nanotopography and material-driven promoting effects. We have grown SHSY5Y human neuroblastoma cells on various substrates and characterized the morphology of the fine cellular processes using HRSEM. The SH-SY5Y cell line has been widely used as a neuronal model in experimental neurological studies on development, degeneration, and differentiation, including Parkinson's disease, Alzheimer's disease, and traumatic brain injury. ${ }^{35}$

To examine whether the positive promoting effect of nanotopography on the neuronal growth can be improved by using active materials as the topographic platform, we have tested the cell growth on substrates coated with AgNPs, a known antibacterial agent. In addition, we have grown the cells on substrates sputtered with a homogenous layer of Ag, with no topographical pattern. Surprisingly, both substrates have led to a significant increased number of neurites emerging from the soma, compared to glass substrates, indicating a material-driven promoting effect on the neurite initiation. Nevertheless, the number of neurites for the AgNPs' substrates is larger than for the sputtered substrates. Measurements of neurites, $5 \mu \mathrm{m}$ from the soma, have revealed no promoting effect for the AgSput substrates, while growth on the AgNPs' substrates has led to an increased number of neurites. This result highlights the physical topography of the substrate as a dominant factor during the neurite growth and elongation phase.

To further investigate the AgNP substrates as a regenerative nanomaterial, we have compared the growth pattern of neuroblastoma cells on the AgNP substrates to substrates that were coated with AuNPs and ZnONPs of similar size and density. We have found a highly significant difference between the Ag coatings and the AuNP and ZnONP coatings for the neurites' initiation phase. Notably, growth on the AgNPs substrates has led to a number of neurites emerging from the soma that is significantly larger than on the AuNP substrates, and on the ZnONP substrates. The nanotopography of all the substrates seems to affect and promote growth with a significant positive maximal effect to the $\mathrm{Ag}$, as reflected in the measured number of neurites $5 \mu \mathrm{m}$ from the soma. Zoomed-in images reveal that the AgNPs function as anchoring sites to which the neurites adhere. The attachment of neurites to the AgNPs induces tensile forces along the neurites and promotes their stabilization, leading to highly straightened neurites. The results of the current study are in agreement with previous work that demonstrated the important role of mechanical tension in neuronal growth and axonal elongation. ${ }^{31-35}$

We have also found that the growth pattern on AgSput substrates is similar to the growth on the glass substrates in the initiation phase, and it was inhibited in the elongation phase, demonstrating no promoting material-driven effect for gold. Thus, we have demonstrated the role of AgNPs as a preferable regenerative substance combining nanotopography and material-driven promoting effects. Usually, the AgNPs are considered cytotoxic. We showed that the AgNPs are not toxic to the cells, confirming recent work by Yen et $\mathrm{al}^{26}$ and Ragaseema et $\mathrm{al}^{27}$ and that they even promote growth. This may be associated with the method we used to produce the NPs. The sonochemical method does not require the addition of stabilizers or other chemicals that may induce cytotoxicity. Other factors that are known to influence cytotoxicity are the biokinetics of NPs, including the rate of their cellular uptake and intracellular distribution. These processes depend on the surface functionalization and the size of the NPs. ${ }^{21}$ The particles used in this study have an average diameter of $110 \mathrm{~nm}$, while most reports on AgNP cytotoxicity use particles in the range of 2-40 $\mathrm{nm}$. Our AgNP-coated substrates contain a relatively low amount of Ag, and the particles are well-attached to the surface. The issue of the leaching of NPs off the glass and other surfaces was studied. Leaving the NP-coated glass in a saline solution for 48 hours did not reveal leaching of the NPs off the surface (data not shown). Therefore, there is no release of NPs or ions $\left(\mathrm{Ag}^{+}\right)$to the medium, which may interact with proteins within cells and cause damage. ${ }^{26}$ Importantly, it has been shown by Perelshtein et $\mathrm{al}^{19}$ and Perkas et $\mathrm{al}^{20}$ that substrates coated with AgNPs, which have similar parameters as the substrates we have used, have a clear antibacterial activity. Thus, the 
AgNP-coated substrates may have a dual activity - both a regenerative effect and an antibacterial effect.

Interestingly, a link between the antibacterial agents and the neural regeneration was previously described in Schikorski et al. ${ }^{36}$ The authors showed that the release of the antibacterial peptides has led to a promoted regenerative effect in an injured central nervous system of the medicinal leech.

\section{Conclusion}

We report the novel use of the AgNPs as a regenerative nanomaterial. The analysis at the single neurite level reveals increased neurite formation and growth, better than on the AuNP and ZnONP substrates or smooth substrates. Thus, we demonstrate a clear material-driven, promoting role for the AgNP substrates, in addition to the nanotopographic effect. Our results, together with the antibacterial properties of AgNPs and the enhanced promoting effect in comparison to other nanomaterials, propose the AgNP substrates as a promising regenerative nanomaterial for neuronal repair applications. In the future, the effect of the AgNPs on the growth of primary neurons should be investigated, testing the regenerative effect on neurons originating from both the central and the peripheral nervous systems. Moreover, using our method enables the fabrication and deposition of AgNPs on different types of surfaces and materials and opens a new avenue of biomedical research in vivo.

\section{Acknowledgments}

We thank Yafit Fleger, Yossi Talyosef, and Yaakov Langzam for their technical assistance with the HRSEM preparation and imaging.

\section{Disclosure}

The authors report no conflicts of interest in this work.

\section{References}

1. Discher DE, Janmey P, Wang YL. Tissue cells feel and respond to the stiffness of their substrate. Science. 2005;310(5751):1139-1143.

2. Théry M, Racine V, Piel M, et al. Anisotropy of cell adhesive microenvironment governs cell internal organization and orientation of polarity. Proc Natl Acad Sci U S A. 2006;103(52):19771-19776.

3. Dowell-Mesfin NM, Abdul-Karim MA, Turner AM, et al. Topographically modified surfaces affect orientation and growth of hippocampal neurons. J Neural Eng. 2004;1(2):78-90.

4. Hanson JN, Motala MJ, Heien ML, Gillette M, Sweedler J, Nuzzo RG. Textural guidance cues for controlling process outgrowth of mammalian neurons. Lab Chip. 2009;9(1):122-131.

5. Mahoney MJ, Chen RR, Tan J, Saltzman WM. The influence of microchannels on neurite growth and architecture. Biomaterials. 2005;26(7): 771-778.

6. Lee JW, Lee KS, Cho N, Ju BK, Lee KB, Lee SH. Topographical guidance of mouse neuronal cell on $\mathrm{SiO}_{2}$ microtracks. Sens Actuators B Chem. 2007;128(1):252-257.
7. Johansson F, Kanje M, Linsmeier CE, Wallman L. The influence of porous silicon on axonal outgrowth in vitro. IEEE Trans Biomed Eng. 2008;55(4):1447-1449.

8. Richert L, Vetrone F, Yi JH, et al. Surface nanopatterning to control cell growth. Adv Mater. 2008;20(8):1488-1492.

9. Pan HA, Hung YC, Sui YP, Huang GS. Topographic control of the growth and function of cardiomyoblast $\mathrm{H} 9 \mathrm{c} 2$ cells using nanodot arrays. Biomaterials. 2012;33(1):20-28.

10. Kang K, Choi SE, Jang HS, et al. In vitro developmental acceleration of hippocampal neurons on nanostructures of self-assembled silica beads in filopodium-size ranges. Angew Chem Int Ed Engl. 2012;51(12): 2855-2858.

11. Prinz C, Hällström W, Mårtensson T, Samuelson L, Montelius L, Kanje M. Axonal guidance on patterned free-standing nanowire surfaces Nanotechnology. 2008;19(34):345101.

12. Xie C, Hanson L, Xie W, Lin Z, Cui B, Cui Y. Noninvasive neuron pinning with nanopillar arrays. Nano Lett. 2010;10(10):4020-4024.

13. Baranes K, Chejanovsky N, Alon N, Sharoni A, Shefi O. Topographic cues of nano-scale height direct neuronal growth pattern. Biotechnol Bioeng. 2012;109(7):1791-1797.

14. Baranes K, Kollmar D, Chejanovsky N, Sharoni A, Shefi O. Interactions of neurons with topographic nano cues affect branching morphology mimicking neuron-neuron interactions. $J$ Mol Histol. 2012;43(4):437-447.

15. Brunetti V, Maiorano G, Rizzello L, et al. Neurons sense nanoscale roughness with nanometer sensitivity. Proc Natl Acad Sci U S A. 2010;107(14):6264-6269.

16. Kim JS, Kuk E, Yu KN, et al. Antimicrobial effects of silver nanoparticles. Nanomedicine. 2007;3(1):95-101.

17. Sharma VK, Yngard RA, Lin Y. Silver nanoparticles: green synthesis and their antimicrobial activities. Adv Colloid Interface Sci. 2009;145(1-2): 83-96.

18. Rai M, Yadav A, Gade A. Silver nanoparticles as a new generation of antimicrobials. Biotechnol Adv. 2009;27(1):76-83.

19. Perelshtein I, Applerot G, Perkas N, Guibert G, Mikhailov S, Gedanken A. Sonochemical coating of silver nanoparticles on textile fabrics (nylon, polyester and cotton) and their antibacterial activity. Nanotechnology. 2008;19(24):245705.

20. Perkas N, Amirian G, Applerot G, et al. Depositing silver nanoparticles on/in a glass slide by the sonochemical method. Nanotechnology. 2008;19(43):435604.

21. Hernández-Sierra JF, Ruiz F, Pena DC, et al. The antimicrobial sensitivity of Streptococcus mutans to nanoparticles of silver, zinc oxide, and gold. Nanomedicine. 2008;4(3):237-240.

22. Raghupathi KR, Koodali RT, Manna AC. Size-dependent bacterial growth inhibition and mechanism of antibacterial activity of zinc oxide nanoparticles. Langmuir. 2011;27(7):4020-4028.

23. Asharani PV, Low Kah Mun G, Hande MP, Valiyaveettil S. Cytotoxicity and genotoxicity of silver nanoparticles in human cells. ACS Nano 2009;3(2):279-290.

24. Asharani PV, Hande MP, Valiyaveettil S. Anti-proliferative activity of silver nanoparticles. BMC Cell Biol. 2009;10:65.

25. Wen HC, Lin YN, Jian SR, et al. Observation of growth of human fibroblasts on silver nanoparticles. J Phys: Conf Ser. 2007;61:445-449.

26. Yen HJ, Hsu SH, Tsai CL. Cytotoxicity and immunological response of gold and silver nanoparticles of different sizes. Small. 2009;5(13):1553-1561.

27. Ragaseema VM, Unnikrishnan S, Kalliyana Krishnan V, Krishnan LK The antithrombotic and antimicrobial properties of PEG-protected silver nanoparticle coated surfaces. Biomaterials. 2012;33(11):3083-3092.

28. Fullenkamp DE, Rivera JG, Gong YK, et al. Mussel-inspired silver-releasing antibacterial hydrogels. Biomaterials. 2012;33(15): 3783-3791.

29. Wright JB, Lam K, Buret AG, Olson ME, Burrell RE. Early healing events in a porcine model of contaminated wounds: effects of nanocrystalline silver on matrix metalloproteinases, cell apoptosis, and healing. Wound Repair Regen. 2002;10(3):141-151. 
30. Tian J, Wong KK, Ho CM, et al. Topical delivery of silver nanoparticles promotes wound healing. Chem Med Chem. 2007;2(1):129-136.

31. Liu X, Lee PY, Ho CM, et al. Silver nanoparticles mediate differential responses in keratinocytes and fibroblasts during skin wound healing. Chem Med Chem. 2010;5(3):468-475.

32. Gedanken A. Using sonochemistry for the fabrication of nanomaterials. Ultrason Sonochem. 2004;11(2):47-55.

33. Buttiglione M, Vitiello F, Sardella E, et al. Behaviour of SH-SY5Y neuroblastoma cell line grown in different media and on different chemically modified substrates. Biomaterials. 2007;28(19):2932-2945.
34. Meijering E, Jacob M, Sarria JC, Steiner P, Hirling H, Unser M. Design and validation of a tool for neurite tracing and analysis in fluorescence microscopy images. Cytometry A. 2004;58(2):167-176.

35. Skotak M, Wang F, Chandra N. An in vitro injury model for SH-SY5Y neuroblastoma cells: effect of strain and strain rate. J Neurosci Methods. 2012;205(1):159-168.

36. Schikorski D, Cuvillier-Hot V, Leippe M, et al. Microbial challenge promotes the regenerative process of the injured central nervous system of the medicinal leech by inducing the synthesis of antimicrobial peptides in neurons and microglia. J Immunol. 2008;181(12):1083-1095. 


\section{Supplementary table}

Table SI Fabrication of substrates coated with zinc oxide nanoparticles and gold nanoparticles

Fabrication of substrates coated with zinc oxide nanoparticles

The $0.55 \mathrm{~g}$ of $\mathrm{Zn}(\mathrm{Ac})_{2}$ was dissolved in $5 \mathrm{~mL}$ of double-distilled water and mixed with $45 \mathrm{~mL}$ of ethanol. A glass slide was positioned in a sonication cell containing $50 \mathrm{~mL}$ of a water-ethanol solution. The solution was then irradiated with a high-intensity ultrasonic horn (Ti horn, $20 \mathrm{kHz}, 600 \mathrm{~W}$ ) for 5 minutes. Then, $0.3 \mathrm{~mL}$ of $25 \mathrm{wt} \%$ aqueous ammonia solution was added to the reaction slurry, and the sonication continued for the following 25 minutes.

\section{Fabrication of substrates coated with gold nanoparticles}

The $0.050 \mathrm{~g}$ of $\mathrm{HAuCl}_{4}(17 \mathrm{wt} \%$ solution in $\mathrm{HCl}$ ) was dissolved in $50 \mathrm{~mL}$ of double-distilled water. A glass slide was positioned in a sonication cell containing a water-ethanol solution, and sonication started. The $0.2 \mathrm{~mL}$ of $25 \mathrm{wt} \%$ aqueous hydrazine hydrate solution was added to the reaction slurry, and the sonication continued for 30 minutes.

\section{Publish your work in this journal}

The International Journal of Nanomedicine is an international, peerreviewed journal focusing on the application of nanotechnology in diagnostics, therapeutics, and drug delivery systems throughou the biomedical field. This journal is indexed on PubMed Central,

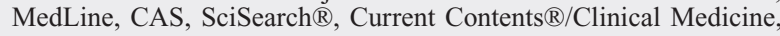

Journal Citation Reports/Science Edition, EMBase, Scopus and the Elsevier Bibliographic databases. The manuscript management system is completely online and includes a very quick and fair peer-review system, which is all easy to use. Visit http://www.dovepress.com/ testimonials.php to read real quotes from published authors.

\footnotetext{
Submit your manuscript here: http://www.dovepress.com/international-journal-of-nanomedicine-journal
} 